\title{
Kinetic and Viscosity Relations for Thermosetting Polyurethanes
}

\author{
M. M. Reboredo, A. J. Rojas, and R. J. J. Williams* \\ Institute of Materials Science \& Technology (INTEMA), \\ University of Mar del Plata, Juan B. Justo 4302, \\ (7600) Mar del Plata, Argentina
}

(Received May 21, 1982)

\begin{abstract}
Kinetic and viscosity relations for polyurethane network polymerization were analyzed. A rigid foam formulation consisting of a polymeric isocyanate (functionality ca. 2.7) and a polyether polyol (functionality $c a .4 .5$ ), was selected. The kinetics were studied with adiabatic temperature rise and chemical techniques. An overall second-order reaction and an activation energy, $E=51.2 \mathrm{~kJ} \mathrm{~mol}^{-1}$, were obtained. The viscosity rise was correlated with the reaction extent and the weight average molecular weight. 'The dependence of viscosity on the latter was of $m$ th order, with $m$ greater than 3.4. This high parametric sensitivity is associated with the formation of branched structures from the beginning of the reaction.

KEY WORDS Thermosetting Polyurethanes / Polyurethane Kinetics / Polyurethane Viscosity / Kinetic-Viscosity Relations / Polyurethane Foams /
\end{abstract}

The processing of thermosetting materials requires a kinetic-viscosity characterization of the system. This is particularly relevant to the analysis of flow through ducts and the filling of molds. ${ }^{1,2}$ The viscosity $(\eta)$ of the polymerizing mixture is best characterized by the weight-average molecular weight $\left(M_{w}\right)$, since both $\eta$ and $M_{w}$ become infinite at the gel point. ${ }^{3}$ On the other hand, an empirical expression relating the viscosity to the extent of reaction $(x)$ and also accounting for the fact that $\eta$ becomes infinite at the gel point $\left(x_{\mathrm{g}}\right)$ is adequate for design purposes. ${ }^{4}$

Several studies on rheological changes during polymerization for polyurethanes have been reported in literature ${ }^{4-7}$ All of them deal with reaction injection molding (RIM) formulations. The purpose of this paper is to present and discuss the results for rigid foam formulations. There is a significant difference between both kinds of formulations which is the average functionality of reactants. Rigid foams are usually made with reactants (one or both) of high functionality to attain a sufficient rigidity at a relatively low reaction extent. That is to say, the gel conversion for foam- ing is lower than for reaction injection molding. This may have a bearing on the rheological behavior. On the other hand, a kinetic-viscosity characterization of a rigid foam formulation might be useful for a detailed analysis of the foaming process.

\section{EXPERIMENTAL}

A typical formulation for rigid foams was selected. The polymeric isocyanate was a polymethylenepolyphenyl isocyanate (PAPI 135, Upjohn), with an average functionality $f_{\mathrm{I}}=2.7$ as reported by the supplier. The NCO content was determined by dissolving a sample in a solution of dibutylamine in toluene and back titrating with $\mathrm{HCl}$ in the presence of bromocresol green (ASTM-D-1638-70). The resulting equivalent weight was $154.7 \mathrm{~g} / \mathrm{eq}$. It was reacted with a polyether polyol based on sorbitol (NIAX LS 490, Union Carbide) with an $\mathrm{OH}$ value of $490 \mathrm{mg} \mathrm{KOH} \mathrm{g}^{-1}$ polyol. The polyol was dehydrated under vacuum for 4 hours at $65^{\circ} \mathrm{C}$. A number-average molecular weight of 521 was determined by cryoscopy in dioxane. The resulting

* To whom all correspondence should be addressed. 
average functionality was $f_{\mathrm{p}}=4.5$.

A reactive mixture was prepared in a tube of $15.5 \mathrm{~cm}$ in length and $7 \mathrm{~cm}$ in internal diameter, and thermally insulated with polyurethane foam. Both reactants were added in stoichiometric amounts at room temperature, and mixing was carried out over 30 seconds with a motor-driven mixer consisting of two propellers with 3 blades each and operating at 2,200 rpm.

Two techniques for measuring the reaction kinetics were used. The first one was the adiabatic temperature rise technique, taking corrections for heat loss into account, ${ }^{8}$ obtained by placing a stopper, with a copper-constantan thermocouple protruding from its center, in the tube where the mixing was performed. The output of the thermocouple was continuously monitored with a data logger (Fluke $2200 \mathrm{~B}$ ). The other technique was based on the chemical determination of the NCO groups which remained unreacted after a certain time at a constant temperature. Several samples wrapped in aluminum foil were placed between two iron plaques maintained at constant temperature. A thermocouple placed in one of the samples showed that temperature was effectively constant. Samples were periodically withdrawn and quenched in a solution of dibutylamine in toluene. The $\mathrm{NCO}$ content was determined by back titrating with $\mathrm{HCl}$ using bromocresol green as the indicator (ASTMD-1638-70).

Viscosity measurements were performed on a Rotovisco RV3 (Haake) using a cone and plate system (PK II: plate diameter $=29 \mathrm{~mm}$; cone diameter $=20 \mathrm{~mm}$; cone angle $=0.00524$ radians). Temperature was controlled by recirculating oil from a thermostated bath (Haake F-3), and measured with a thermocouple located in the plate (a special hole was drilled in the plate for this purpose). Small sample sizes and long reaction times ensured that non-isothermal effects due to heat of reaction would be negligible.

\section{KINETICS}

Figure 1 shows the experimental temperature rise (solid line) in the quasi-adiabatic reactor. The dotted line is the adiabatic temperature calculated from the experimental curve, taking corrections for heat loss into account. ${ }^{8}$ The adiabatic temperature, $T$, verifies the following energy balance:

$$
c_{\mathrm{p}} \mathrm{d} T / \mathrm{d} t=(-\Delta H) \mathrm{d} x / \mathrm{d} t
$$

where $c_{\mathrm{p}}$ is the specific heat and $(-\Delta H)$ is the heat evolved per unit mass, both assumed constant.

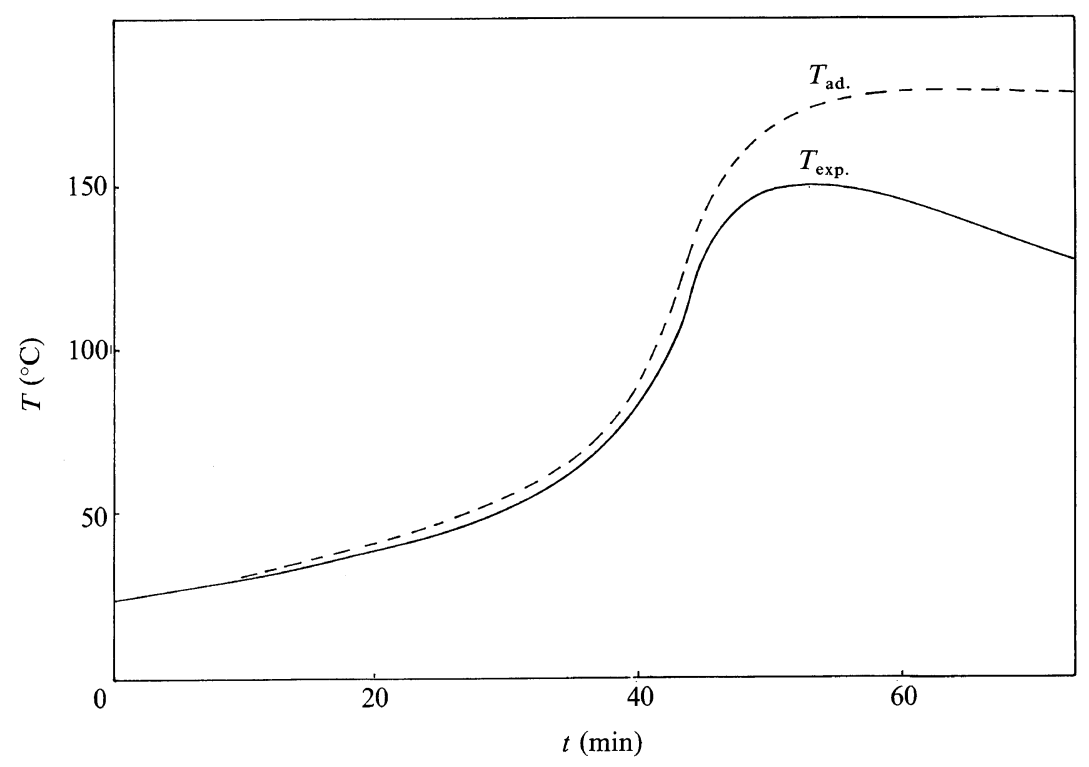

Figure 1. Experimental temperature rise (solid line) and calculated adiabatic temperature rise (dotted line). 
From equation 1, the conversion is given by

$$
x=\left(T-T_{0}\right) / \Delta T_{\mathrm{ad}}
$$

where $\Delta T_{\mathrm{ab}}=(-\Delta H) / c_{\mathrm{p}}$. If an $n$th order kinetics is proposed to fit the adiabatic curves,

$$
\mathrm{d} x / \mathrm{d} t=A(1-x)^{n} \exp (-E / R T)
$$

Substituting with equations 2 and 3 , equation 1 was rearranged as

$$
\begin{aligned}
\ln k & =\ln \left\{\frac{\mathrm{d} T / \mathrm{d} t}{\Delta T_{\mathrm{ad}}\left[1-\left(T-T_{0}\right) / \Delta T_{\mathrm{ad}}\right]^{n}}\right\} \\
& =\ln A-E / R T
\end{aligned}
$$

The $n$ value which gives a correlation coefficient (c.c.) close to unity when making the linear regression, was the one selected. $A$ and $E$ arise from the corresponding ordinate and slope. An excellent regression (c.c. $=0.9975$ ) was obtained for $n=2$, as shown in Figure 2. The kinetic parameters took the following values:

$$
A=5.87 \times 10^{4} \mathrm{~s}^{-1}, \quad E=51.2 \mathrm{~kJ} \mathrm{~mol}^{-1}
$$

Both the reaction order and the activation energy are in good agreement with the results reported for reaction injection molding formulations. ${ }^{9}$

A comparison between the adiabatic temperature rise method and the chemical technique for determining the kinetics is shown in Figure 3. Points are the experimental results of the extent of the reaction as a function of time at $35^{\circ} \mathrm{C}$, obtained with the chemical technique. The curve is the prediction of the kinetic expression arising from the adiabatic temperature rise method. The agreement is very satisfactory up to conversions close to 0.3 . No accurate results at $x>0.3$ could be obtained, due to the fact that as the conversion approached the gel point (see following section), the samples became difficult to dissolve in a short period of time.

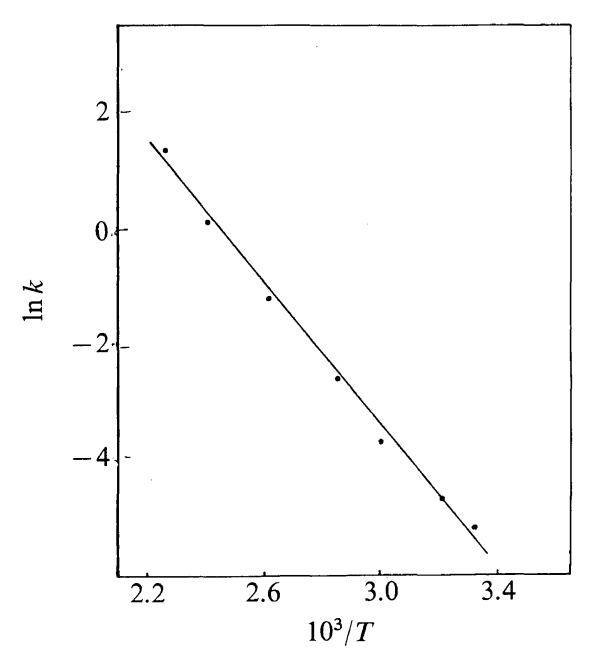

Figure 2. Linear regression according to equation 4 for an overall second-order reaction.

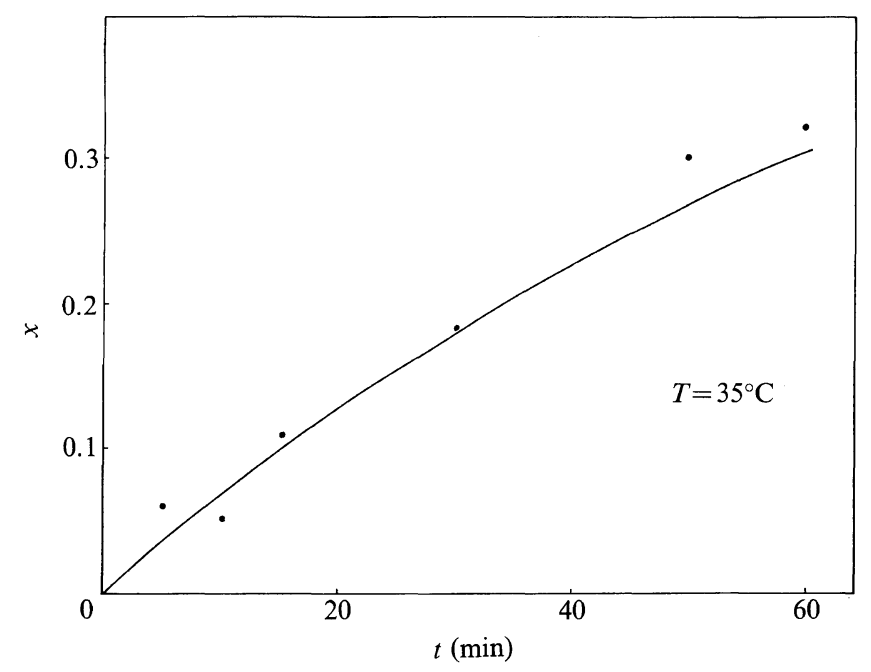

Figure 3. Extent of the reaction as a function of time, at $35^{\circ} \mathrm{C}$. Points are the experimental results obtained with the chemical technique. The cruve is the prediction of the kinetic expression arising from the adiabatic temperature rise method. 


\section{THE GEL CONVERSION}

An estimation of the gel conversion may be obtained by assuming that the reactants have narrow distributions of functionalities. In this case, ${ }^{10}$

$$
x_{\mathrm{g}}=1 /\left\{\left(f_{\mathrm{I}}-1\right)\left(f_{\mathrm{P}}-1\right)\right\}^{1 / 2}=0.41
$$

An approximate experimental gel point was obtained by pulling the thermocouple gently during quasi-adiabatic reaction, and quoting the temperature value at which the thermocouple resisted to move. Several runs gave $x_{\mathrm{g}}$ values in the range $0.35-0.40$; in reasonable agreement with the theoretical prediction. For subsequent calculations, an $x_{\mathrm{g}}=0.40$ was adopted.

\section{VISCOSITY}

The viscosity $v s$. time curves obtained at $32.5^{\circ} \mathrm{C}$, $40.5^{\circ} \mathrm{C}$, and $50.1^{\circ} \mathrm{C}$, are shown in Figure 4. Two runs carried out at the same temperature showed reasonable reproducibility. For the first part of the curves, where the rate of the slope increase was not so high, measurements were periodically made over

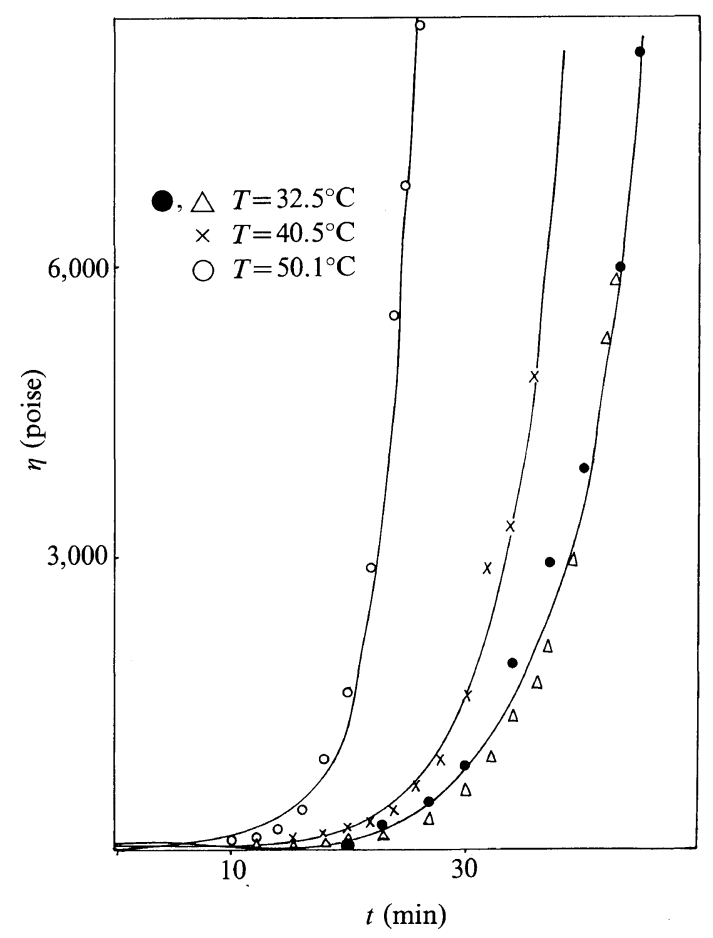

Figure 4. Viscosity vs. time at $32.5,40.5$, and $50.1^{\circ} \mathrm{C}$. a range of shear rates during a short period of time. No significant shear thinning was observed, which is in agreement with the results reported for RIM formulations. ${ }^{5,6}$

\section{KINETIC-VISCOSITY RELATIONS}

Using the kinetic expression (eq 3), viscosity vs. time curves may be transformed into viscosity vs. conversion curves. The results are plotted in Figure 5 as $\ln \eta / \eta_{0}$ vs. $x$. A significant feature is that the dependence on temperature is not very important, a fact that has also been reported for RIM formulations..$^{4,7}$ As shown in the figure, experimental data are roughly fitted with the following simple equation:

$$
\eta / \eta_{0}=\left\{x_{\mathrm{g}} /\left(x_{\mathrm{g}}-x\right)\right\}^{7.5}
$$

with $x_{\mathrm{g}}=0.40$. Thus, with these high-functionality reactants, viscosity shows a very high parametric sensitivity toward the reaction extent. This effect may be very important for stabilizing the bubbles formed in the course of a foaming process at a low

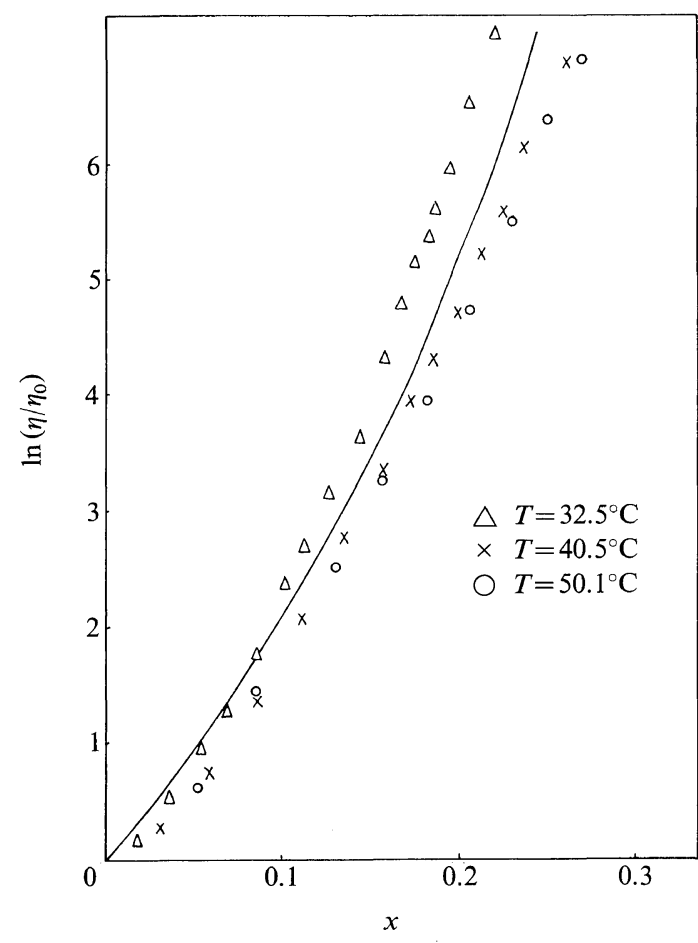

Figure 5. Relative viscosity $v s$. fractional conversion at various temperature. 


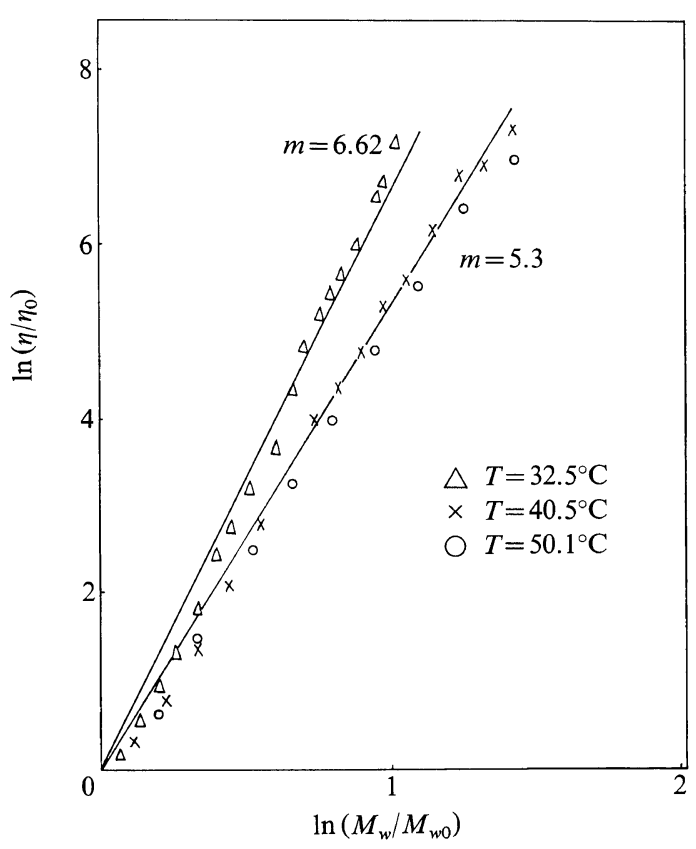

Figure 6. Relative viscosity vs. weight-average molecular weight at various temperature.

reaction extent.

In order to find a correlation of viscosity with the weight average molecular weight, $M_{w}$, it is necessary to relate the last quantity with the fractional conversion, $x$, using the network polymerization theory. By assuming that both the isocyanate and polyol are monodisperse reactants, an assumption which proved to be reasonable for the gel conversion estimation, the following equation was obtained ${ }^{10}$ :

$\frac{M_{w}}{M_{w 0}}=1+\frac{x f_{\mathrm{P}}\left[x\left(f_{\mathrm{P}}-1\right) M_{\mathrm{I}}^{2}+x\left(f_{\mathrm{I}}-1\right) M_{\mathrm{P}}^{2}+2 M_{\mathrm{I}} M_{\mathrm{P}}\right]}{M_{w 0} M_{\mathrm{P}}\left(1+m_{\mathrm{I} 0} / m_{\mathrm{P} 0}\right)\left[1-x^{2}\left(f_{\mathrm{P}}-1\right)\left(f_{\mathrm{I}}-1\right)\right]}$

$M_{w 0}$ is the initial weight-average molecular weight, $M_{\mathrm{I}}$ and $M_{\mathrm{P}}$ are the molecular weights of the isocyanate and polyol, and $m_{\mathrm{I}} / m_{\mathrm{PO}}$ is the initial mass ratio.

Figure 6 shows a plot of the relative viscosity, $\eta /$ $\eta_{0}$, as a function of $M_{w} / M_{w 0}$, in a double logarithmic scale. For a large number of linear polymers,

$$
\eta / \eta_{0}=\left(M_{w} / M_{w 0}\right)^{m}
$$

where $m$ varies from 1 for low molecular weight species to about 3.4 for long entangled chains. ${ }^{11}$
Previously reported studies on rheological changes during network polymerizations ${ }^{6}$ showed $m$ values to be in the range 1 to 2.6. In our case, $m$ took on values greater than 3.4, as shown in Figure 6. This high sensitivity of viscosity toward the weight-average molecular weight must be due to the high functionality of the reactants. It is worthy to point out that $m$ values greater than 3.4 may also be obtained when phase separation takes place during the viscosity rise. $^{7}$

\section{CONCLUSIONS}

A kinetic-viscosity relation was obtained for a polyurethane formulation for rigid foams. Average functionalities were 2.7 for the isocyante and 4.5 for the polyol. An overall second-order kinetics, with an activation energy of $E=51.2 \mathrm{~kJ} \mathrm{~mol}^{-1}$, was obtained with a quasi-adiabatic temperature rise method, taking corrections for heat loss into account. The kinetic expression was verified by performing isothermal runs and following the reaction extent with a chemical technique. The relative increase in viscosity showed a high parametric sensitivity on reaction extent, a fact that might be important in the stabilization of bubbles produced in the foaming process. Viscosity showed an $\mathrm{m}$ th order dependence on the weight-average molecular weight, with $m$ values greater than 3.4. This unique high sensitivity of viscosity on conversion and weight-average molecular weight may be explained by the high functionality of both the reactants, leading to branched structures from the very beginning of the reaction.

Acknowledgments. The authors gratefully acknowledge the support of this work by the Comisión de Investigaciones Científicas de la Provincia de Buenos Aires (CIC), Argentina. Fellowship support from the CIC(MMR) is also acknowledged. Two of the authors (AJR and RJJW) are Research Members of the Consejo Nacional de Investigaciones Científicas y Técnicas, Argentina.

\section{REFERENCES}

1. A. J. Rojas, H. E. Adabbo, and R. J. J. Williams, Polym. Eng. Sci., 21, 634 (1981).

2. J. M. Castro and C. W. Macosko, Studies of mold 
filling and curing in the reaction injection molding (RIM) process, AIChE J., in press.

3. P. J. Flory, J. Am. Chem. Soc., 63, 3091 (1941).

4. J. M. Castro and C. W. Macosko," Rheology of Polyurethane Reaction Injection-Molding Systems," Proceedings of 3rd International Meeting on Polymer Science and Technology, La Plata, Argentina, 1981, p 276.

5. S. D. Lipshitz, F. G. Mussatti, and C. W. Macosko, SPE ANTEC Tech. Papers, 21, 239 (1975).

6. S. D. Lipshitz and C. W. Macosko, Polym. Eng. Sci., 16, 803 (1976).
7. J. M. Castro, F. López Serrano, R. E. Camargo, C. W. Macosko, and M. Tirrell, J. Appl. Polym. Sci., 26, 2067 (1981).

8. A. J. Rojas, J. Borrajo, and R. J. J. Williams, Polym. Eng. Sci., 21, 1122 (1981).

9. E. B. Richter and C. W. Macosko, Polym. Eng. Sci., 18, 1012 (1978).

10. C. W. Macosko and D. R. Miller, Macromolecules, 9 , 199 (1976).

11. T. G Fox and G. C. Berry, Adv. Polym. Sci., 5, 261 (1968). 